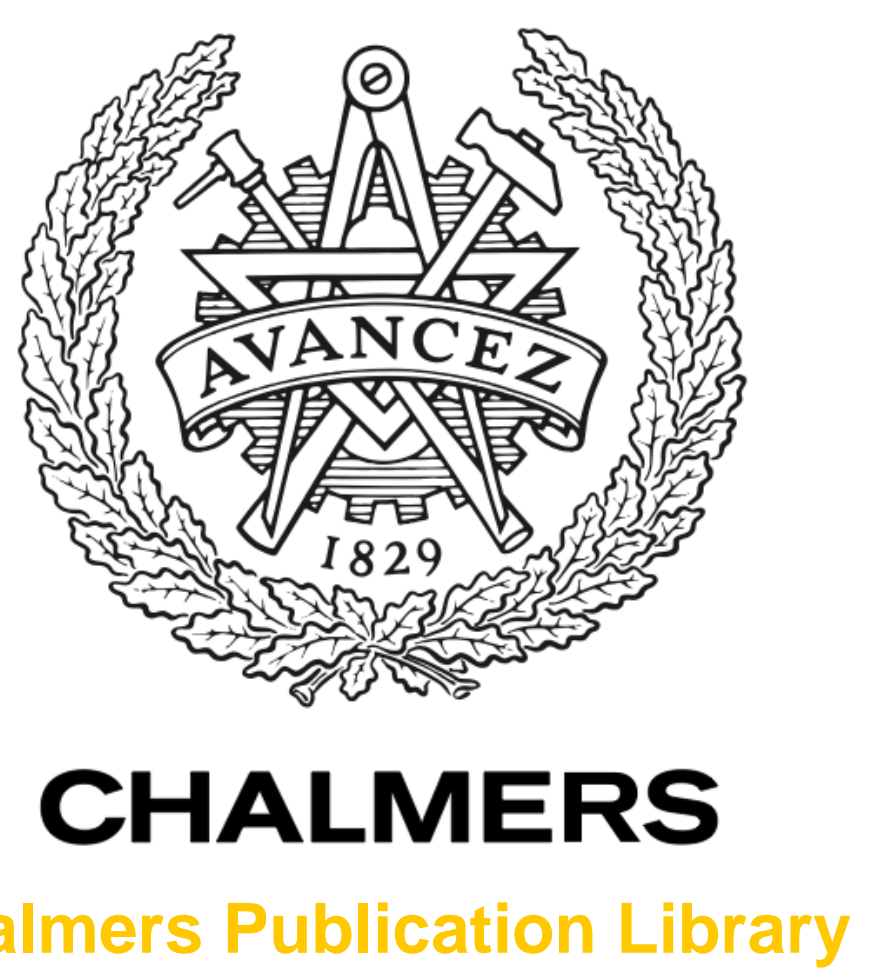

Chalmers Publication Library

\title{
Vertically illuminated TW-UTC photodiodes for terahertz generation
}

This document has been downloaded from Chalmers Publication Library (CPL). It is the author's version of a work that was accepted for publication in:

Proceedings of SPIE - The International Society for Optical Engineering. Millimeter, Submillimeter, and Far-Infrared Detectors and Instrumentation for Astronomy VI, Amsterdam, 3-6 July 2012 (ISSN: 0277-786X)

Citation for the published paper:

Barrientos Z, C. ; Calle, V. ; Alvarez B, J. (2012) "Vertically illuminated TW-UTC photodiodes for terahertz generation". Proceedings of SPIE - The International Society for Optical Engineering. Millimeter, Submillimeter, and Far-Infrared Detectors and Instrumentation for Astronomy VI, Amsterdam, 3-6 July 2012, vol. 8452

http://dx.doi.org/10.1117/12.927440

Downloaded from: http://publications.lib.chalmers.se/publication/172033

Notice: Changes introduced as a result of publishing processes such as copy-editing and formatting may not be reflected in this document. For a definitive version of this work, please refer to the published source. Please note that access to the published version might require a subscription. 


\title{
Vertically Illuminated TW-UTC Photodiodes for Terahertz Generation
}

\author{
Claudio M. Barrientos Z. ${ }^{1}$, Victor H. Calle G. ${ }^{1}$, Jaime A. Alvarez ${ }^{1}$ B., F. Patricio Mena ${ }^{1}$, Josip \\ Vukusic $^{2}$, Jan Stake ${ }^{2}$, and Ernest A. Michael ${ }^{*}$ \\ ${ }^{1}$ Terahertz Photonics Laboratory, Department of Electrical Engineering, University of Chile, \\ Santiago de Chile \\ ${ }^{2}$ Department of Microtechnology and Nanoscience MC2, Chalmers University of Technology \\ Gothenburg, Sweden \\ *Corresponding author: emichael@ing.uchile.cl
}

\begin{abstract}
More efficient and powerful continuous-wave photonic mixers as terahertz sources are motivated by the need of more versatile local oscillators for submillimeter/terahertz receiver systems. Uni-Travelling Carrier (UTC) photodiodes are very prospective candidates for reaching this objective, but so far only have been reported as lumped-elements or as edge-illuminated optical-waveguide travelling-wave (TW) devices. To overcome the associated power limitations of those implementations, we are developing a novel implementation of the UTC photodiodes which combines a travelingwave photomixer with vertical velocity-matched illumination in a distributed structure. In this implementation called velocity-matched travelling-wave uni-travelling carrier photodiode, it is possible to obtain in-situ velocity matching of the beat-fringes of the two angled laser beams with the submm/THz-wave on the stripline. In this way, minimum frequency roll-off is achieved by tuning the angle between the two laser beams. A first design of these TW-UTC PDs from our Terahertz Photonics Laboratory at University of Chile has been micro-fabricated at the MC2 cleanroom facility at Chalmers Technical University.
\end{abstract}

Keywords: photomixer, traveling wave, laser mixing, sub-millimeter, terahertz, radiation source, local oscillator

\section{INTRODUCTION}

DEVELOPMENT of terahertz frequency systems has been widely studied during the last years, leading to a wide range of applications in several fields like astronomy, biology, communications, imaging, medicine and security. For radio astronomy applications more efficient continuous-wave $(\mathrm{cw})$ photonic mixers as terahertz sources are being developed with the main motivation to fabricate more versatile photonic local oscillators for $\mathrm{THz}$ radio astronomical receivers. An important application would be high-resolution and -precision molecular spectroscopy of the many space-relevant molecules yet still to be measured for interpreting data of ALMA (higher resolution means lower confusion limit) and space projects (e.g. Herschel).

To overcome the power limitations of lumped element and travelling-wave edge-coupled devices, a top illuminated travelling-wave (TW) scheme is applied, similar to previously investigated LT-GaAs based photomixers [2][11]. In this configuration the travelling-wave photomixing idea is integrated with two others concepts, (i) large-area vertical illumination with adjustable velocity matching, and (ii) a uni-travelling carrier layer scheme for ultrafast photodiodes (UTC-PD) [4]. The final device, called vertically-illuminated velocity-matched travelling-wave uni-travelling carrier photodiode (VM TW-UTC PD), has been partially simulated, modelled and optically tested in our Terahertz Photonics Laboratory at the Electrical Engineering Department of the University of Chile, whereas device micro-fabrication was performed at the MC2 clean room facility at Chalmers University of Technology.

This paper describes the design, micro-fabrication process and very first experimental results of a first batch of these devices designed to operate at $0.2-2 \mathrm{THz}$. The main goal behind the fabrication and testing of this novel type of

Millimeter, Submillimeter, and Far-Infrared Detectors and Instrumentation for Astronomy VI, edited by Wayne S. Holland, Jonas Zmuidzinas, Proc. of SPIE Vol. 8452 ,

84522I @ 2012 SPIE · CCC code: 0277-786X/12/\$18 · doi: 10.1117/12.927440

Proc. of SPIE Vol. $8452845221-1$ 
photomixer is to achieve a higher power/efficiency-bandwidth product, and for our purpose of application as a local oscillator in radio astronomy that means mainly higher power at high frequencies.

\subsection{Travelling-Wave Photodiodes}

The principal advantage of travelling-wave photomixers is avoiding the parasitic RC time constant limitation of the corresponding lumped element. This means that bandwidth and power can be improved at the same time using a travelling-wave technique [1].

This is different from lumped-element mixers, where an optimization between minimization of device capacity for reaching higher speed, and maximization of device area for maximization of input power (and thus capacity) exists. By using travelling-wave devices this trade-off is bypassed and it is feasible to improve simultaneously bandwidth and input power capacity. Additionally, bandwidth and output power are favoured by the factor $\left(1+\omega^{2} \tau_{R C}^{2}\right)$ [1], [2].

Travelling-wave photomixers can be divided in two main classes depending of the effective absorption coefficient of the devices structure [10], low effective absorption $\alpha \ll 1 \mu \mathrm{m}^{-1}$ leads to edge-coupled (fibre-illuminated) photomixing, whereas high absorption $\alpha \gg 1 \mu m^{-1}$ leads to top illuminated large-area designs with velocity-matching.

Other groups are developing edge-illuminated devices [15], containing a special coupling scheme from the optical waveguide to the distributed photodiode, which achieves responsivities of $0.5 \mathrm{~A} / \mathrm{W}$ [15]. However, due to a certain damage threshold intensity at the input facet and due to the small optical cross section, the power which can be coupled to the input facet was not reported yet much higher than $50 \mathrm{~mW}$.

\subsection{Uni-Traveling Carrier Photodiodes}

For increasing the $\mathrm{THz}$ power at higher frequencies, the carrier transit time must be minimized. The most efficient photomixers to date in generating submillimter radiation up a terahertz were shown to be Uni-travelling Carrier Photodiodes (UTC-PD) [4], because they restrict the hole transport to just near one electrode, and let the electrons transit a collection layer ballistically, so that the effective transit-time is reduced very much.

In transit-time dominated devices, like p-i-n or UTC photodiodes, the recombination time is much longer than the transit time. Therefore, the minimization of the transit time should result in an only minor reduction of the dc-photocurrent. Before UTC photodiodes where invented, the fastest photodiodes for high frequency applications were p-i-n photodiodes, with a $3 \mathrm{~dB}$-frequency in the range of $50 \mathrm{GHz}$.

Since the maximal achievable output power also depends on the pump power capacity, larger diode areas are desirable. However, increasing the diode cross section for lumped-element photomixers the RC time constant worsens, so that a trade-off exists between power capability and speed.

In lumped-element UTCs, the $R_{A} C$ constant is improved by a larger electrode distance by inserting a transparent (larger-gap) collection layer in which the electrons are believed to move at least partially ballistic and so are not contributing significantly to the total transit time $\tau_{\text {trans }}=\tau_{A}+\tau_{C}$, and $\tau_{C}<\tau_{A}$.

Thus, using the UTC-PD concept, bandwidth and output saturation current are increased in comparison to p-i-n photodiodes and therefore higher output power can be obtained at higher frequencies [3],[4]. Bandwidths up to 300 GHz were shown [7].

\subsection{Response of a Photomixer}

In the photomixing process, the output power at low input powers is proportional to the square of the photocurrent, at higher powers saturation should occur. Therefore we assume [17]:

$$
P_{T H z} \propto P_{N I R}^{2} \cdot \exp \left(-A \cdot P_{N I R}\right)
$$

In which $\mathrm{A}$ is to be determined. For an ideally velocity-matched TW photomixer, illuminated by two single mode $\mathrm{CW}$ lasers beams, the THz output power should be described approximately according to [4] and [12]:

$$
P_{T H z}(\omega)=\frac{R_{A} I_{e, 0}{ }^{2}}{2\left[1+\left(\omega \tau_{e}\right)^{2}\right]}
$$

because the RC time constant, giving an additional roll-off factor of $\propto 1 /\left(1+(\omega R C)^{2}\right)$, is bypassed . 
$R_{A}$ is the antenna impedance, $I_{e 0}$ is the DC value of the electronic photocurrent resulting at the beat frequencies of both lasers, and $\tau_{e}$ is the photoelectron response time, which is the mean time from the generation of the photoelectron to a current signal at the electrodes. It is assumed that the stripline is impedance matched to the antenna.

For a first assessment, $\tau_{e}$ can be regarded as a unique, unpartitioned time constant. However, in case of the UTC photodiode structure, several authors propose to separate this time into two time-constants [4], [12]: $\tau_{e}=\tau_{A}+\tau_{C}$, the mean diffusion time from the absorption layer to the interface to the collection layer $\tau_{A}$, and the travel time through the collection layer $\tau_{C}$. This comes in when the electron and hole currents are averaged over the diode structure thickness, with the motivation to make accessible the instantaneous voltage across the photodiode structure and therefore derive a correct expression for the frequency response. This approach introduces then an additional factor describing interference effects from the coherent ballistic transit of the photoelectrons through the collection layer, once generated in the absorption layer,

$$
P_{T H Z}(\omega)=\frac{I_{e, 0}^{2} R_{A}}{2\left[1+\left(\omega \tau_{A}\right)^{2}\right]} \times\left|\frac{\sin \left(\omega \tau_{C} / 2\right)}{\omega \tau_{C} / 2}\right|^{2}
$$

which would represent an additional roll-off at lower frequencies, and a spectral oscillation including sharp blankings of any output at higher frequencies. $\tau_{A}=W_{A} / v_{t h}+W_{A}^{2} / 2 D_{e}$ is the mean time of the photoelectrons from generation to the interface absorption and collection layer. $W_{A}$ is the thickness of the absorption layer, $D_{e}=(k T / q) \cdot \mu_{e}(E, N)$ is the diffusion coefficient, in there $k$ is the Boltzmann constant and $T$ is the temperature. The mobility $\mu_{e}(E, N)$ is a function of the electric field strength $E$ and the doping concentration $N$.

The carrier transit time $\tau_{C}$ would then depend on how the electrons perform the transit through the collection layer, ballistically or drifting. In reality, it is expected to be a mixture of both. The ballistic time is $\tau_{C}=(2 s / a)^{0.5}=\left(2 m_{e} / e V\right)^{0.5} s$, and for $\mathrm{s}=150 \mathrm{~nm}$ and $\mathrm{V}=2 \mathrm{~V}$ we have $\tau_{C}=5 \cdot 10^{-13} \mathrm{~s}$. The frequency above which this term would be relevant is $300 \mathrm{GHz}$. Experimentally this interference effect has not yet been observed in edgecoupled TW-devices [4][15][15][7]. The observation of a smooth roll-off between $f^{-2}$ and $f^{-3}$, up to above $1 \mathrm{THz}$ points more towards frequency-dependent absorption and/or a partial velocity mismatch. We expect that our results would also contribute to the assessment of that theory. The DC-photocurrent is given by:

$$
I_{e, d i f f, 0}=R\left(V, P_{N I R, 0}\right) \cdot P_{N I R, 0}
$$

where $R\left(V, P_{N I R, 0}\right)$ is the bias and input power saturation dependent DC-responsivity, which depends also on the geometry of the photomixer. We are conducting numerical calculations in Synopsys TCAD to provide that function, and plot the responsivity curves for a simple geometry (see paragraph ...).

\section{VERTICALLY ILLUMINATED TW-UTC PHOTOMIXER DESIGN AND MODELING}

\subsection{Concept of Top Illuminated Velocity-Matched Travelling-Wave Photomixing}

To achieve highest input power levels, as $P_{T H z} \propto P_{N I R}{ }^{2}$, as long as there is no saturation, we are investigating a vertical angled illumination scheme for a TW-UTC-PD which is capable, by virtue of the large optical cross section, of very high input power levels. In fact, we could observe a power capability of our novel TW-photomixers (see Fig. 1) in excess of $500 \mathrm{~mW}$.

Moreover, the vertical angled illumination scheme (see Fig. 1 and 2) is capable of in-situ matching of the optical group velocity (interference fringes) to the phase velocity of the to be generated terahertz wave on the metal stripline. This illumination and phase-matching scheme was demonstrated first by Matsuura et al. [2][3], and was developed further and verified for MSM-type LT-GaAs TW-photomixers [9].

The concept of the vertical illumination velocity matched UTC-PD (VM TW-UTC-PD) is depicted in Fig. 1. 


\section{NEW CONCEPT:}

\section{Uni-travelling carrier photodiode (UTC-PD)

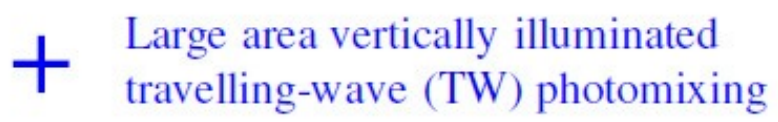

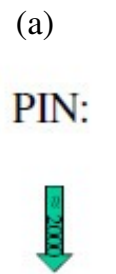

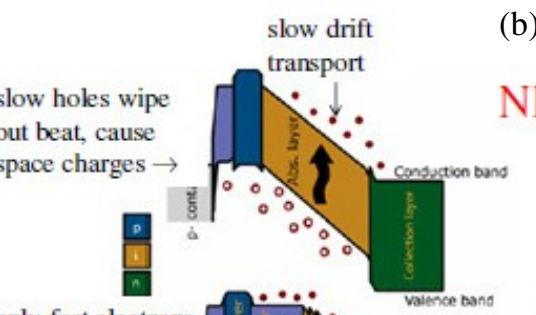

(b)

UTC: $\begin{aligned} & \text { only fast electron } \\ & \text { bridge gap } \rightarrow\end{aligned}$

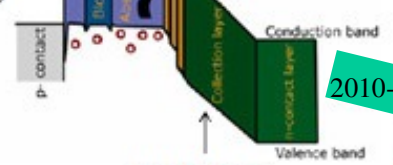

fast ballistic

transport

\section{$=\quad \mathrm{VMTW}$ TWTC}

Fig. 1: (a) p-i-n-PD versus UTC-PD. (b) Integration with vertically illuminated velocity matched travelling-wave concept. The difference frequency of the two stripline foci of the lasers creates a running interference fringe pattern that produces a photocarrier density wave travelling along the strip-line (see Fig. 2).

\subsection{Vertical angled illumination}

In the case of velocity-matched top illumination, also called angle-tuned phase matching [2][3][10][11], a velocity matching is realized by adjusting the angle between the two lasers, and therefore the velocity of the interference fringes along the surface of the stripline. This is explained in Fig. 2a.

(a)

$$
f_{N I R, 1} \downarrow \downarrow f_{N I R, 2}<f_{N I R, 1}
$$
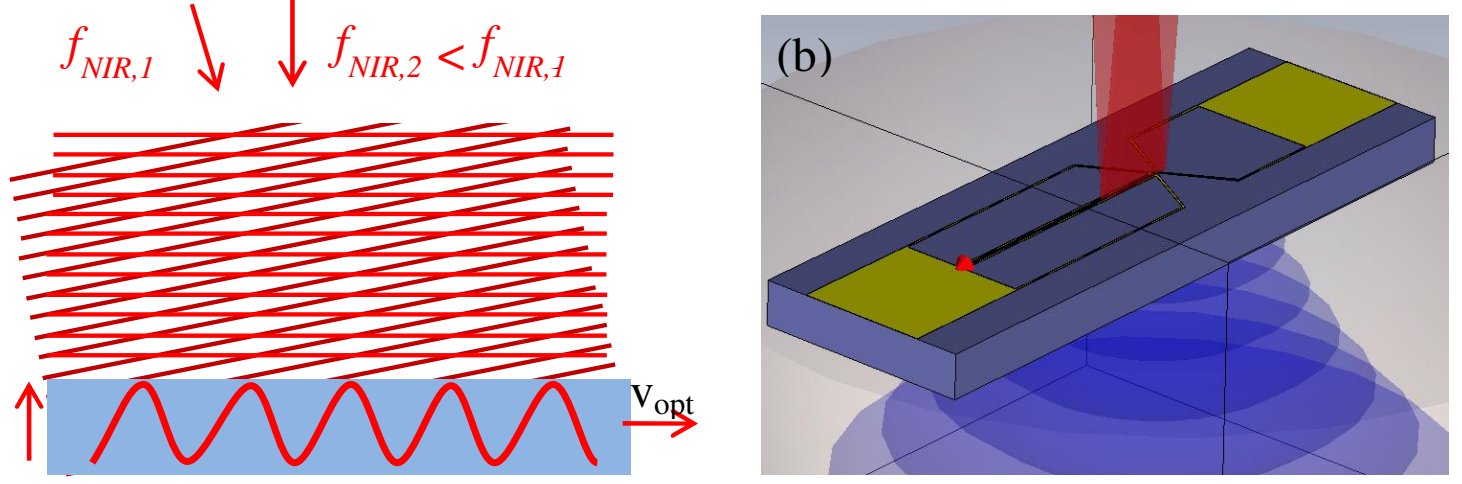

Fig. 2: (a) Principle of generating the running fringes under angled mixing of the two laser beams. (b) Conceptual view of the mixer chip on top of the Si-lens with the angled NIR-beams and the THz-wave radiated into the substrate. The large angular divergence of the individual NIR-beams is better shown in Fig. 3. 


$$
v_{\text {opt }}=2 \pi \frac{f_{1}-f_{2}}{k_{1, \|}-k_{2, \|}}=\frac{c\left(f_{1}-f_{2}\right)}{f_{1} \sin \left(\theta_{1}\right)-f_{2} \sin \left(\theta_{2}\right)} \approx \frac{c}{\left(1+f_{2} / f_{T H z}\right) \sin (\theta)}
$$

where $f_{2}$ is the frequency of the fixed, lower frequency laser and $f_{T H z}=f_{1}-f_{2}$ is the THz-frequency. The THz-wave velocity is $v_{T H z}=c / \sqrt{\varepsilon_{e f f}}$, so that the phase mismatch is of the form $\Delta k=\omega\left(v_{T H z}{ }^{-1}-v_{o p t}{ }^{-1}\right)$ (see [2]). The maximal attainable THz-power for a phase-mismatch would be described by:

$$
P_{T H z}=P_{T H z, \max } \cdot F(\Delta k)
$$

where $F(\Delta k)<1$ for $\Delta k \neq 0$ and $F(\Delta k)=1$ for $\Delta k=0$.

Depending on the substrate thickness the effective dielectric constant goes over from $\varepsilon_{\text {eff }}=(\varepsilon-1) / 2$ at low frequencies to $\varepsilon_{\text {eff }}=\varepsilon$ at high frequencies [5]. Taking into account the attached hemispherical Si-lens, in addition to the chip thickness of $0.3 \mathrm{~mm}$, we have very large substrate thickness compared to the THz-wavelength, so that we have clearly the second case, other than assumed in [2]. Especially there should be no dispersion of the THz-wave velocity and so there was never observed any profile of the function through any deviation from the $\mathrm{f}^{2}$ roll-off more significant than could be explained by increase of the absorption with frequency.

Therefore we could build an optics scheme in which the velocity-match is built into for automatic tuning of the angle, independently on the frequency. For this, it is employed a grating from where the two laser frequencies enter the following cylinder-lens based optics with an angle between them proportional to the frequency difference of the two laser beams, and therefore to the THz-frequency [11].

If the beam profiles are simplified to be rectangular, one has according to [2]:

$$
F(\Delta k)=\frac{\sin ^{2}(\Delta k L / 2)}{(\Delta k L / 2)^{2}}
$$

For Gaussian beam profiles the response is less oscillating in the wings at $|\Delta k L / 2| \gg 1$ than given by this, but of similar width of the main maximum [9].

\subsection{Focussing on the mesa stripline of the CPW waveguide}

InGaAs has a high absorption coefficient of $\alpha \approx 1.8 \mu \mathrm{m}^{-1}$ at $0.8 \mathrm{eV}(1.55 \mu \mathrm{m})$, therefore it is a reasonable apploach to treat UTC-PDs within the vertical illumination class of photomixers. However, the thickness of the absorption layer $\mathrm{W}_{\mathrm{A}}$ is necessary to be kept thin $\left(\mathrm{W}_{\mathrm{A}}<150 \mu \mathrm{m}\right)$ to have a fast device [4]. Therefore the absorbed power fraction is only 1 $\exp (-\alpha \cdot 2 \mathrm{WA}) \approx 0.35$ (see Fig. 3, internal reflection from metal stripline), corresponding to a responsivity of $0.44 \mathrm{~A} / \mathrm{W}$, if assuming complete focusing of the beam into the central stripline of $4 \mu \mathrm{m}$. This can be achieved, since with $\mathrm{f}=6.5 \mathrm{~mm}$ and NA $=\mathrm{D} / 2 \mathrm{f}=0.4$ of the last focusing lens, about the maximum NA available for a diffraction-limited lens, we obtain with $w_{0}=\lambda / \pi \theta, \theta=w_{\text {coll }} / 2 f$ and $w_{\text {coll }} \approx D / 2 \ldots D / 3$, a waist of $w_{0}=(2 \ldots 3) \times \lambda / \pi N A=1.55 \ldots 2.32 \mu m$, so that the power fraction collected in $4 \mu \mathrm{m}$ stripline width is 0.99 . Given a small central blockage by the CPW (see Fig. 3), we expect about $0.4 \mathrm{~A} / \mathrm{W}$. However, entering the substrate, Snell's law transforms to a much smaller convergence, so that the waist becomes larger approximately by the factor of the refractional index of $n=3.5$, giving a power overlap with the stripline of just 0.54 .

Fig. 3: Focusing scheme: The two NIR-beams are reflected from a NIR-mirror at the back-side of the chip, either dielectric surface reflection maintaining a certain gap (30\%), a Bragg-reflector $(100 \%)$, or a grid of gold-strips $(80 \%$, which is at the same time a THz-polarization filter). See text.

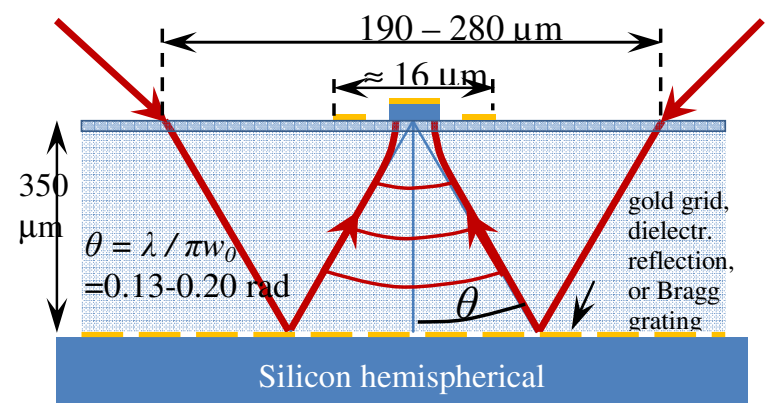


Unfortunately, at the end of our first successful fabrication run the grid back reflector did not work out. Altogether, this results in a much reduced illumination efficiency: 0.3 times from the reflector and 0.5 times due to the mismatch of the beam-waist. But in spite of these deficiencies we can still test the devices using the pump geometry of Fig. 3, since we have a $1.55 \mu \mathrm{m}$ fiber power amplifier with an output power up to $5 \mathrm{~W}$. Giving 0.15 coupling efficiency, and $50 \%$ grating transmission, the effective input power to the mixers would be up to $300 \mathrm{~mW}$. For the final devices we plan to fabricate transparent electrodes on top of the center mesas from Indium tin oxide (ITO) known to be a good reflector (low losses) at least in the submm-range, together with a Bragg-reflector directly under the subcollector layer.

\subsection{TW-UTC Photomixer Design and Modeling}

\section{a) Coplanar Waveguide (CPW) design}

In order to hold the radiation and substrate mode losses low at higher frequencies, the total transversal width $\mathrm{w}$ of the waveguide should be as small as possible. Further, the gap should represent a low series resistance for for the photocurrents, so $c$ should be small. However, the characteristic impedance of the CPW becomes low when the gap is reduced. This is unwanted since a broadband matched antenna would be less radiation effective. Therefore, also to allow fabrication tolerances (very large aspect ratio of the waveguide featrue), the gap spacing was chosen to $3 \mu \mathrm{m}$. The center stripline width $a$ should not be too small since the sharpest diffraction-limited optical focusing gives a focus waist of $\mathrm{w}_{0}$ $=1.5 \mu \mathrm{m}$, and this should give a high overlap with the center stripline (mesa width). The critical fabrication steps for the airbridge require definition of a photoresist opening less in width than this stripline. Therefore $4 \mu \mathrm{m}$ were the utmost minimum, and was indeed very demanding to fabricate. Since the characteristic impedance tend to be very low (order of $30 \Omega$ ), $b$ should be as small as possible to raise it as much as possible, but sufficient DC-current capacity should still be given.

\section{b) Antenna design:}

Different types of antennas such as logarithmic spiral and log-periodic structures have been reported [4][7], but the impedance of those broadband antennas $(\approx 70 \Omega)$ is relatively low for our purpose. Since terahertz power is proportional to the antenna impedance, higher-impedance antennas are desirable, but cannot be realized with a broadband match to the CPW. A true slot bow-tie antenna has a much higher impedance compared to its inverse metal bow-tie antenna, according to $Z_{\text {slot }}=Z_{0}^{2} / Z_{\text {metal }}$. It would be above $1000 \Omega$, too high for a matching to any CPW.

A stripline bow-tie antenna has been designed together with the CPW waveguide to reduce the reflections at the footpoint of the antenna and so minimize standing waves (fig. 1). This type of antenna, basically a wire-antenna, displays an impedance similar to the CPS.

\section{MODELING AND SIMULATION}

\subsection{Electromagnetic Modeling}

The electromagnetic performance of the scheme shown in figure 2 was obtained after optimization using CST Microwave Studio and HFSS. Whereas the first device was designed on reasonable thinking, we intent to include the results of the current simulations and measurements to design an optimized device for the second fabrication run.

The $S_{11}$ parameter which is better than around $-10 \mathrm{~dB}$ for the frequency range so and so shows resonances due to the presence standing waves which are consequences of the impedance mismatch between the CPW and the slot bow-tie antenna. 


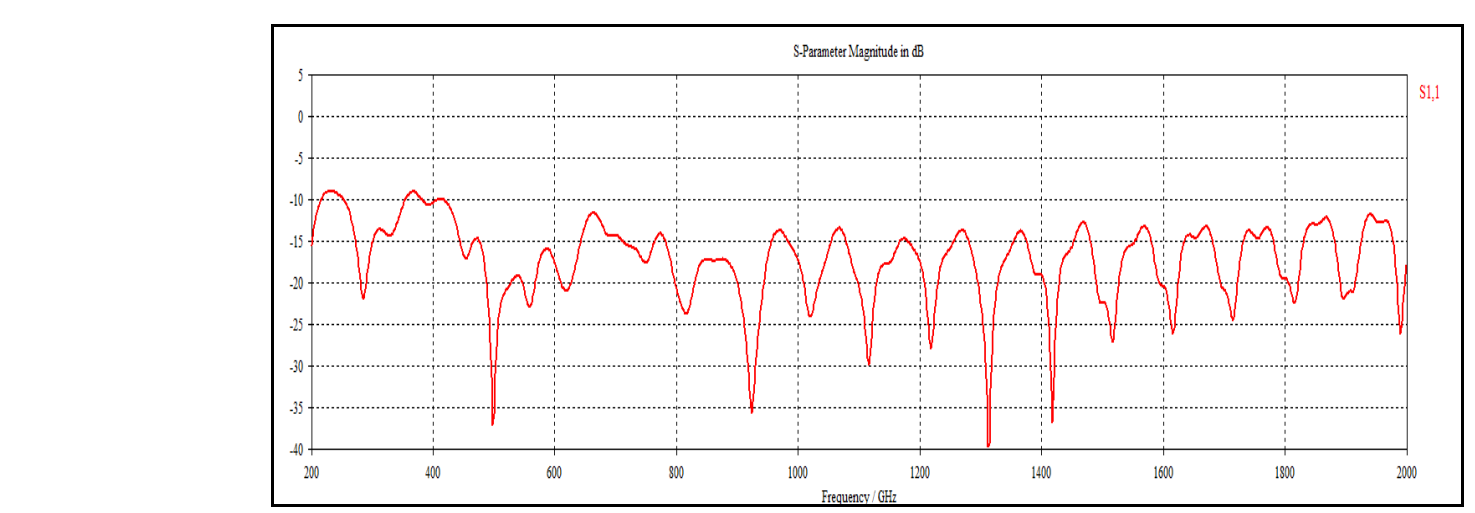

Fig. 5: $\mathrm{S}_{11}$ parameter for slot bow-tie antenna with $60^{\circ}$ of aperture angle and 350 um length

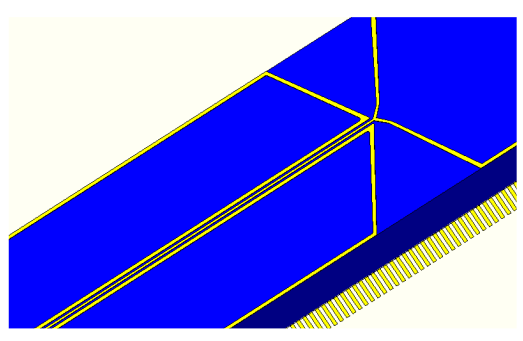

Device shape in CST Microwave Studio. Under the structure a grid was placed to work as a IR mirror and $\mathrm{THz}$ polarization filter.
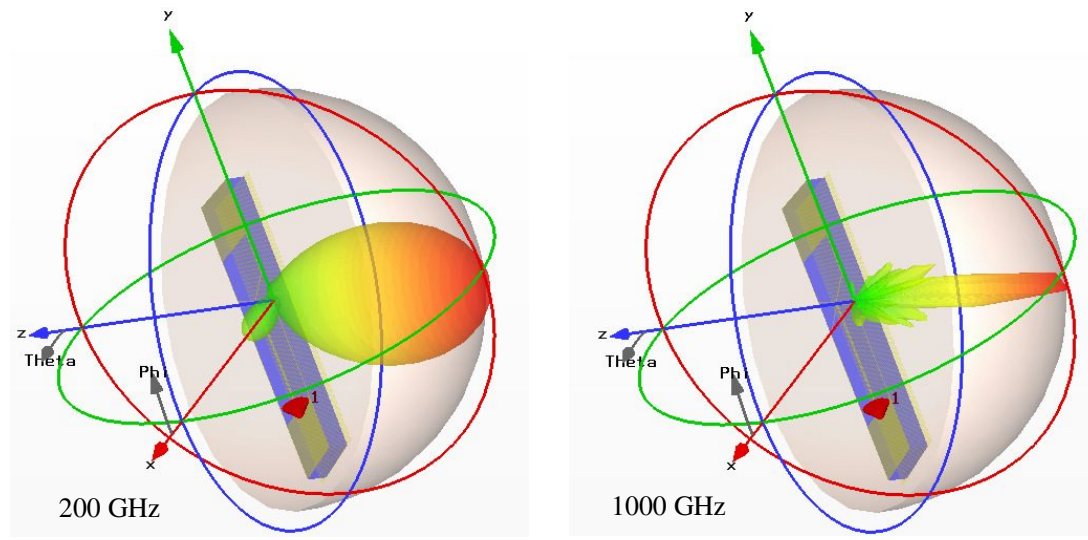

Fig. 6: Farfield patterns calculated for $200 \mathrm{GHz}$ and $1000 \mathrm{GHz}$.

\subsection{TCAD Simulation of the Layer Structure}

We are developing simulations in Synopsys TCAD, based on previous work of Banik et al. [13], and Mahmudur et al. [14] in order to elucidate how to improve the layer structure with respect to minimum possible transit times and maximum responsivities, regardless of the capacity, since that would be not effective. However, the literature gives discussion of both, drift and overshoot ballistic movement of the electrons through the collection (transport) layer. [12].

\section{FABRICATION OF THE TW-UTC PHOTOMIXERS}

To fabricate our InGaAs/InP TW UTC-PD's, standard III-V clean room micro-processing techniques are used. This includes UV photolithography, e-beam lithography, e-beam metal evaporation deposition; contact annealing, wet and dry etching, sputtering, electroplating and ion beam milling. Additionally, the back-side is lapped and polished, in order to have an optical surface for the transmission of the submm-beam and the reflection of the NIR-beam for backillumination of the photodiode because the central stripline is in-transparent. Later also an ITO-based contact would be tried for the latter for more diffraction limited front side illumination, but the advantage of the gold is that the beam focused from the back over the bottom NIR-mirror (see fig. 3) is passing the absorption region $\mathrm{W}_{\mathrm{A}}$ twice. Then also a Bragg-grating is considered to be embedded under the vertical structure. Since the device is top illuminated, a $200 \mathrm{~nm}$ layer of $\mathrm{Si}_{3} \mathrm{~N}_{4}$ is intended to be deposited on the top side as an anti-reflection coating.

For the first chip iteration, the layer structure of our device was based in the optimization performed in [13], the details of the epitaxial layer structure are shown in Table 1 . Notice that layer 12 represents the p-contact on the photodiode, and layer 3 the n-contact, in order to fabricate a p-ohmic contact a layering of $\mathrm{Ni} / \mathrm{Au} / \mathrm{Pt} / \mathrm{Ti} / \mathrm{Pt}$ (3000/2000/800/300/100 $\AA$ ) was done. The Ni layer on top is only applied to work as a mask for the dry etching step during which it is greatly reduced; a process of inverse reversal photolithography and descum was performed. The metal 
contact was evaporated using a UHV e-beam evaporator, after lift-off the p-contact was annealed at $320^{\circ} \mathrm{C}$ by 30 s. The first chain of those steps is graphically represented in Fig. 7.

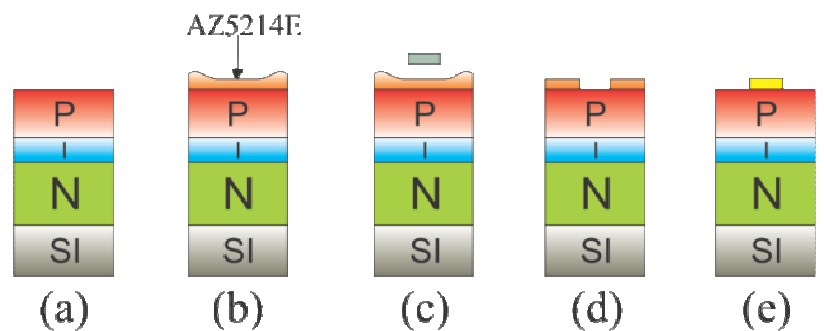

Fig. 7: In this figure the UTC layer structure is represented by that of a p-i-n photodiode. (a) whole substrate, (b) substrate after photoresist coating, (c) a quartz photo mask is used to create the p-contact (d) photoresist shape after developing and descum, (e) pcontact after lift-off and stripping process. As the last step during p-contact fabrication annealing was used to improve the adhesion between the metal and the semiconductor surface.

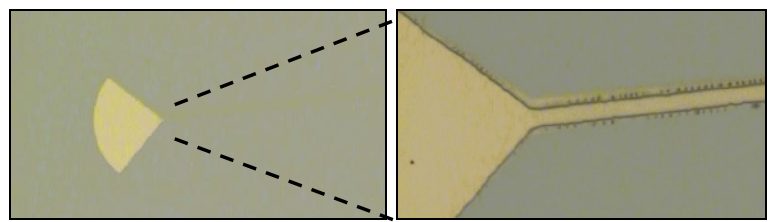

Fig. 8: Microscope picture of the p-contact. The picture shows the central conductor of the coplanar-wave guide that works as pcontact. The triangular shape works as attenuator for backward waves.

TABLE 1: Used UTC layer system.

\begin{tabular}{|l|l|l|l|}
\hline LAYER & MATERIAL & THICKNESS [nm] & DOPING \\
\hline 12 & $\mathrm{In}_{0.53} \mathrm{Ga}_{0.47} \mathrm{As}$ & 50 & $3 \mathrm{e} 19 \mathrm{p}+$ \\
\hline 11 & $\mathrm{In}_{0.53} \mathrm{Ga}_{0.47} \mathrm{As}_{0.80} \mathrm{P}_{0.20}$ & 20 & $2 \mathrm{e} 19 \mathrm{p}+$ \\
\hline 10 & $\mathrm{In}_{0.53} \mathrm{Ga}_{0.47} \mathrm{As}$ & $125\left(=\mathrm{W}_{\mathrm{A}}\right)$ & $1 \mathrm{e} 18 \mathrm{p}$ \\
\hline 9 & $\mathrm{In}_{0.53} \mathrm{Ga}_{0.47} \mathrm{As}$ & 8 & $1 \mathrm{e} 15 \mathrm{i}$ \\
\hline 8 & $\mathrm{In}_{0.76} \mathrm{Ga}_{0.24} \mathrm{As}_{0.54} \mathrm{P}_{0.28}$ & 16 & $1 \mathrm{e} 15 \mathrm{i}$ \\
\hline 7 & $\mathrm{InP}$ & 6 & $1 \mathrm{e} 15 \mathrm{i}$ \\
\hline 6 & $\mathrm{InP}$ & 7 & $1 \mathrm{e} 18 \mathrm{n}$ \\
\hline 5 & $\mathrm{InP}$ & $150\left(=\mathrm{W}_{\mathrm{C}}\right)$ & $1 \mathrm{e} 16 \mathrm{n}-$ \\
\hline 4 & $\mathrm{InP}$ & 50 & $5 \mathrm{e} 18 \mathrm{n}$ \\
\hline 3 & $\mathrm{In}_{0.53} \mathrm{Ga}_{0.47} \mathrm{As}$ & 10 & $1 \mathrm{e} 19 \mathrm{n}+$ \\
\hline 2 & $\mathrm{InP}$ & 500 & $>2 \mathrm{e} 19 \mathrm{n}+$ \\
\hline 1 & $\mathrm{In}_{0.53} \mathrm{Ga}_{0.47} \mathrm{As}$ & 10 & $\mathrm{I}$ \\
\hline Sub & $\mathrm{InP} \mathrm{SI}$ & & $\mathrm{SI}$ \\
\hline
\end{tabular}

In order to fabricate the CPW mesa structure, a $\mathrm{Cl}_{2} / \mathrm{H}_{2} / \mathrm{CH}_{4}$-based inductively coupled plasma (ICP) process has been applied using the Ni-layer as the dry etch mask. The dry etching was carried out in an Oxford Plasmalab, until half of layer 4, after that selective wet etching is used until layer 3, where the ohmic n-contact of Pt/Ti/Au is deposited.

Once the dry etching step is reached, in order to avoid any dark current between both cathodes through the n-doped layers 2 and 1, a pattern mask and a positive photoresist is used to isolate the upper contact pad with the bottom contact, once the pattern is transferred to the wafer, selective wet etching of InGaAs and $\mathrm{InP}$ is performed since the photoresist acts as a mask, the upper part is etched until the SI layer. This way the n-contact and the p-contact connected to the upper contact pad through the air-bridge are isolated. 


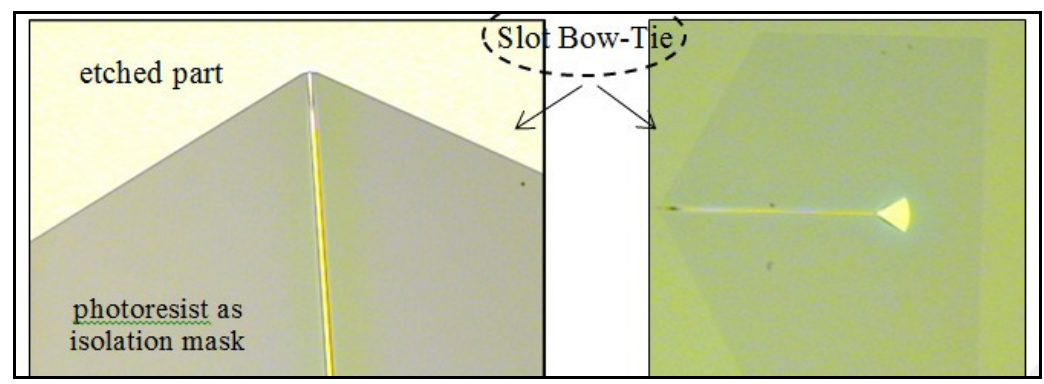

Fig. 9: Photoresist is used as an etching mask to protect the region where the n-contact is deposited. In the etched part the opposite side of the antenna is deposited later.

(a)

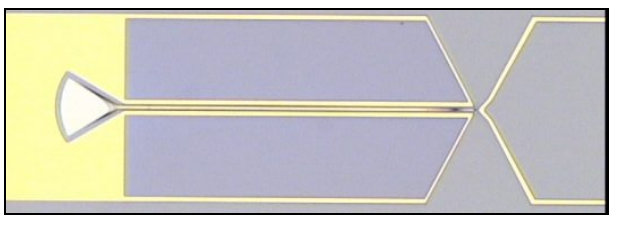

(b)

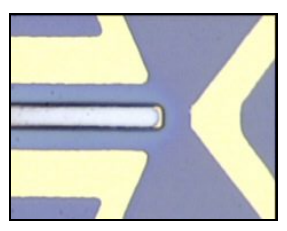

Fig. 10: Bow-tie slot antenna integrated to a CPW on the device. (a) whole TW-UTC-PD device (except right contact pad), (b) zoom of the CPW structure ( $\mathrm{p}$ and $\mathrm{n}$ contacts)

After the isolation step, the n-contact is built. To fabricate this contact, an inverse reversal photoresist is used as in the case of the p-contact. The n-contact has the shape shown in Fig. 4.

The next step is the fabrication of the air-bridge which is used to electrically connect the upper contact pad with the p-contact. The air-bridge was fabricated in four main process steps: e-beam photolithography, sputtering, electroplating and ion-milling.

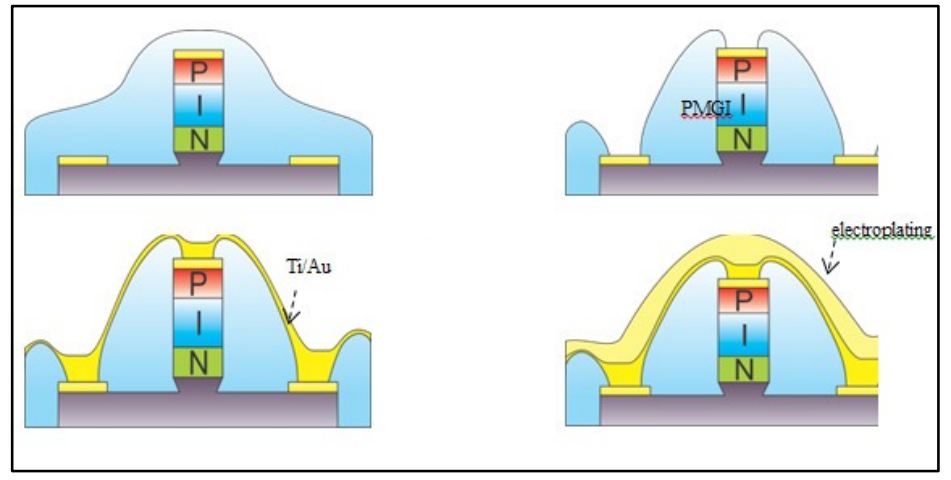

Fig. 11: The fabrication of the air-bridge is performed in four main steps. 1) PMGI e-beam-resist is coated on the chip. 2) A pattern is used to open areas on the p-contact and $\mathrm{n}$-contact. 3) A thin layer of Ti/Au is sputter and after that electroplating is performed.4) Ionbeam milling is used to remove the metal in the parts where the metal was not grown.
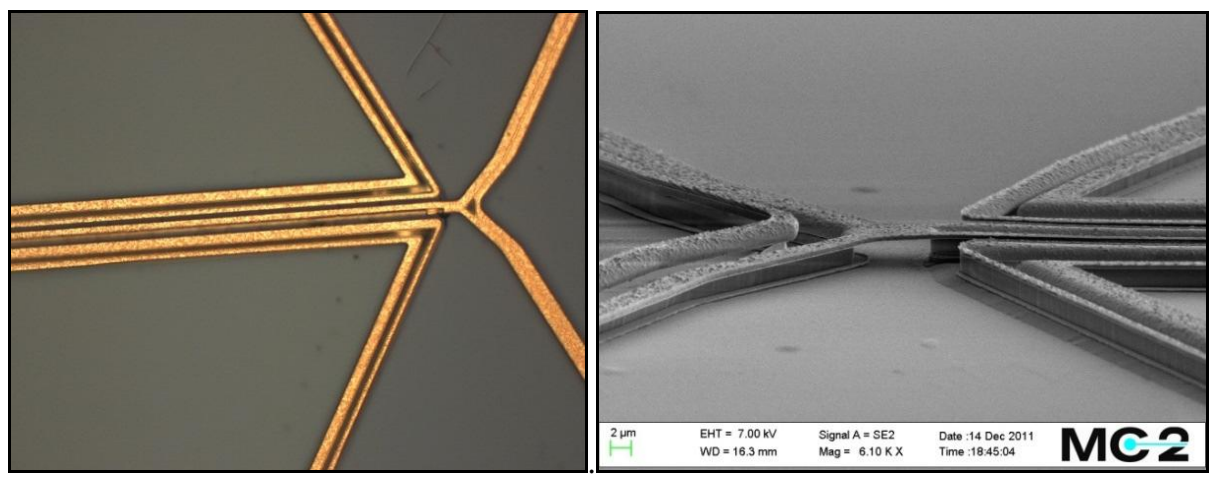

Fig. 12: Left panel: A microscope picture of the structure with the air-bridge. Right panel: a SEM picture of the air-bridge. 


\section{EXPERIMENTAL SETUP}

The optical experiment set-up is shown in Fig. 13. As we mention before the device is top illuminated by two lasers with a difference frequency $\Delta f$, which can be adjusted between 0 and several THz (1 THz is $\lambda=8 \mathrm{~nm} @ 1550 \mathrm{~nm})$. The two lasers, entering through a common fiber and collimating fiber to free-space coupler, pass through to a cylindrical lens with a focal length of $80 \mathrm{~mm}$, which produces a vertical stripline focus on the grating. Both laser beams are split by a blazed grating to produce an angle difference between the two laser colors, and the resultant beams are collimated by a second cylinder lens. A third cylinder lens focuses them again into two vertical stripline foci, the separation of which are translated by the last, aspheric lens into an angle between the beams on the photomixer. This separation can be adjusted by the separation between the second and third cylinder lens, and so the angle. In the vertical plane, which was not modified by all the cylindrical lenses, the beams are focused diffraction limited by the aspheric lens, while in the horizontal direction the aperture of this lens is not fully illuminated and therefore the focus is unsharp (the long stripline). At the end of the system two long spots of $300 \mu \mathrm{m}$ length and $4 \mu \mathrm{m}$ width are obtained with an angle difference of $2.4^{\circ}$. The optics were simulated with the ray-tracing program Zemax.
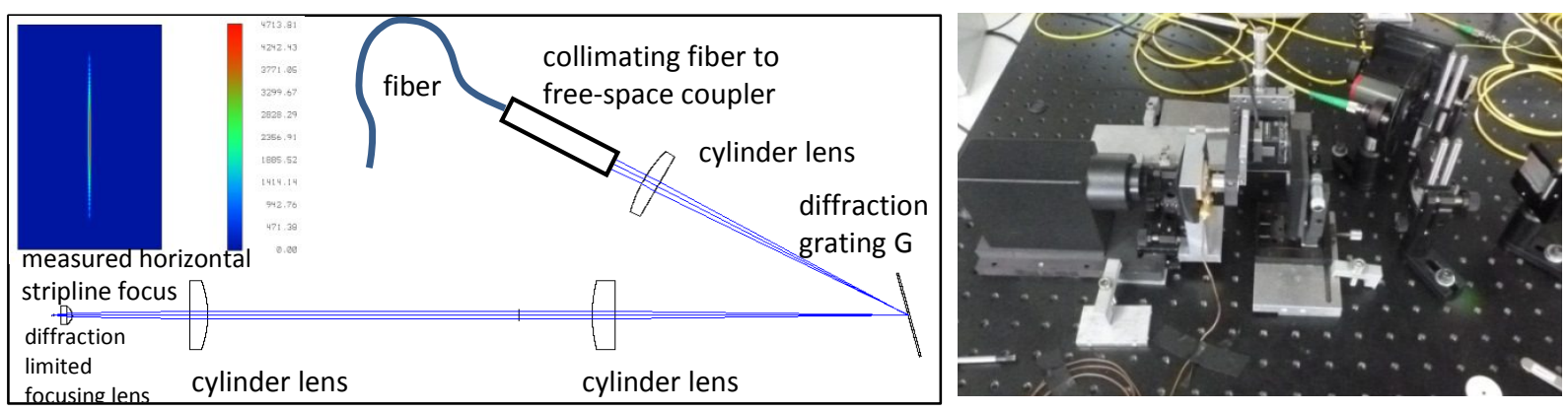

Fig. 13: Left panel: Zemax simulation for the optical system. Right panel: Picture of the experimental set-up.

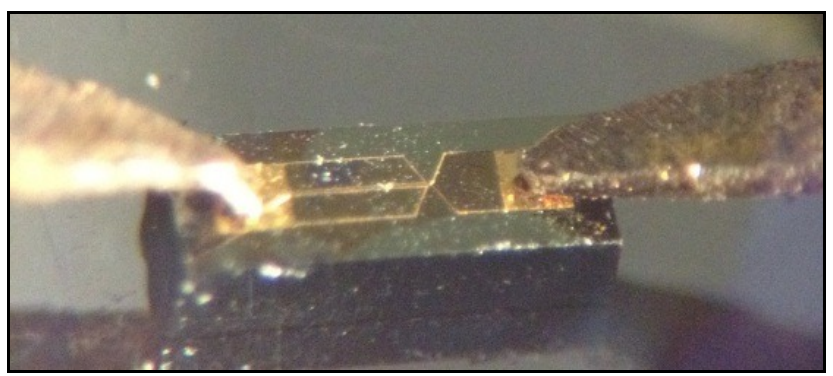

Fig. 14: Photograph of a chip pressed down onto a Si-lens surface by contact pins. The size of the antenna is $300 \mu \mathrm{m}$.

After some batches could be finished successfully the first time end of last year, first experimental results were expected to be available before this conference but due to various technical problems this could not be achieved.

\section{ACKNOWLEDGEMENT}

We acknowledge support from the ALMA-Conicyt funds $\mathrm{N}^{\circ} 31080020$ and 31110014 for Chilean Astronomy, from Fondecyt fund $\mathrm{N}^{\circ}$ 1090306, and from the ALMA project for the loan of some decommissioned pre-production components.

\section{REFERENCES}

[1] K.S. Giboney, M.J.W. Rodwell, and J.E. Bowers, "Traveling-Wave Photodetector Theory," IEEE Trans. Microwave Theory and Techiques 45, p. 1310-1319 (1997)

[2] S. Matsuura, G. A. Blake, R. A. Wyss and J. C. Pearson, C. Kadow, A. W. Jackson, and A. C. Gossard, "A traveling-wave THz photomixer based on angle-tuned phase matching," Appl. Phys. Lett. 74, p. 2872 (1999) 
[3] S. Matsuura et al., "Free-space Travelling-Wave Terahertz Photomixers," Proc. of the IEEE 7. Int. Conference on THz Electronics, Nara, Japan, p. 24, (1999)

[4] T. Ishibashi et al., "Uni-travelling carrier photodiodes," Tech. Dig. Ultrafast Electronics and Optoelectronics, Incline Village NV USA, p. 83-87 (1997)

[5] C. Fumeaux, W. Herrmann, F.K. Kneubühl, H. Rothuizen, "Nanometer thin-film Ni-NiO-Ni diodes for detection and mixing of $30 \mathrm{THz}$ radiation," Infrared Physics \& Technology 39, p. 123-183 (1998)

[6] T. Ishibasi, T. Futura, H. Fushimi, S. Kodama, H. Ito, T. Nagatsuma, N. Shimizu, anf Y. Migamoto, "InP/InGaAs Uni-Travelling-Carrier Photodiodes," IEICE Transaction on Electronics E83-C, p. 938-49 (2000)

[7] H. Ito, T. Furuta, S. Kodama and T. Ishibashi, "InP/lnGaAs uni-travelling-carrier photodiode with $310 \mathrm{GHz}$ bandwidth," Electronics Letters 36, p. 1809 (2000)

[8] S. Matsuura, and H. Ito, "Generation of CW Terahertz Radiation with Photomixing," Topics in Applied Physics, Springer Berlin, 97, p. 157-202 (2006)

[9] E. A Michael, M. Mikulics, M. Marso, Peter Kordoš, H. Lüth, B. Vowinkel, R. Schieder, and J. Stutzki, "Largearea travelling-wave LT-GaAs photomixers for LO application," Proc. of the SPIE Astronomical Instrumentation Glasgow Scotland 5498, p. 525 (2004)

[10] E. A Michael, "Travelling-wave photonic mixers for increased continuous-wave power beyond $1 \mathrm{THz}$," Semiconductor Science and Technology 20, p. 164-177 (2005)

[11] E. A. Michael, M. Mikulics, B. Vowinkel, R. Schieder, M. Marso, and P. Kordoš, "Large-area traveling-wave photonic mixers for increased continuous terahertz power," Appl. Phys. Lett. 86, 111120 (2005)

[12] M. N. Feiginov, "Analysis of limitations of terahertz vp-i-n uni-traveling-carrier photodiodes," J. of Appl. Phys. 102,084510 (2007)

[13] B. Banik, J. Vukosic, H. Hjelmgreen, and J. Stake, "Optimization of the UTC-PD Epitaxy for Photomixing at 340 GHz," Int. Journal of Infrared and Millimeter Waves, p. 914-923 (2008)

[14] S.M. Mahmudur Rahman, H. Hjelmgren, J. Vukusic, J. Stake, P. A. Andrekson, and H. Zirath, "Hydrodynamic Simulations of Unitraveling-Carrier Photodiodes," IEEE J. Quantum Electronics 11, p. 1088 (2007)

[15] E. Rouvalis et al., "A Travelling-Wave Uni-Travelling Photodiode for Continuous Wave Terahertz Generation," London Communications Symposium, ... (2009)

[16] E. Rouvalis et al., "Continuous Wave Terahertz Generation From Ultra-Fast InP-Based Photodiodes," IEEE Transactions on Microwave Theory and Techniques 60, p. $509-517$ (2012)

[17] E. A. Michael and M. Mikulics, "Losses from long-living photoelectrons in terahertz-generating continuous-wave photomixers,” App. Phys. Lett. 100, 191112 (2012) 